\title{
特別寄稿
}

\section{The relationship between subjective and objective measures of nasal function}

\author{
Ronald Eccles \\ Common Cold Centre, School of Molecular and Medical Biosciences \\ Cardiff University Cardiff CF1 3US United Kingdom
}

Key words : nasal sensation of airflow, nasal congestion, rhinomanomery, acoustic rhinometry, menthol

\begin{abstract}
Sensations from the nose (symptoms) are more important to the patient than objective measures of nasal function obtained by rhinomanometry and acoustic rhinometry. In order to understand nasal symptoms, the definitions of terms such as 'nasal congestion', ' nasal obstruction' and ' nasal sensation of airflow' are put forward for discussion. The concept of 'nasal sensation of airflow' as measured by a visual analogue scale has helped in the understanding of the effects of menthol on the nose and demonstrated that there is no relationship between the objective and subjective effects of menthol on the nose. Studies on patients have found little or no relationship between objective and subjective measures of nasal function when both nasal passages are considered together, and only a poor relationship when the nasal passages are studied separately. Progress in this area is dependent on standardising the methods and terminology used for subjective measures of nasal function and a better understanding of the factors that influence the patients perception of nasal disease.
\end{abstract}

\section{INTRODUCTION}

The sensory nerve supply to the nose and upper airway is important in the control of breathing (1) and in the protection of the airway from inhalation of noxious substances and gases $(2,3)$ but these aspects of neurophysiology which have been mainly studied in animal models will not be discussed in the present review. The present review will focus on the sensations from the human airway in health and disease which can be described and measured experimentally.

Nasal sensations are important in the study of nasal disease as it is the patients perception of nasal sensations (symptoms) rather than changes in nasal function which is of primary concern to the patient. However, the clinician in the assessment and treatment of nasal disease is mainly concerned with restoring normal function to the nasal airway by altering nasal airway resistance either by medical or surgical means. The clinician has various objective methods of assessing the nasal airway such as rhinomanometry and acoustic rhinometry but the problem is that these objective measures of nasal function do not always correlate with the patients own assessment of nasal sensations such as the sensation of nasal airflow.

The controversy and confusion in this area is demonstrated by two recent publications; one which claims to demonstrate a correlation between nasal airway resistance and nasal sensation of airflow and which concludes that rhinomanometry provides useful information about the symptom of nasal obstruction (4) ; and the other which reports that there is no correlation between measurements of nasal resistance and nasal sensation of airflow, and that the rhinomanometer has little clinical value (5).

There is also much confusion in the literature as 
there has been no standardisation of the terms used to describe and measure nasal sensations and research papers in this area give very little or no information about the methods used to quantify nasal sensations.

The present review will focus on the symptom often described as ' nasal obstruction' related to diseases of the nose.

A major problem in this area concerns the terminology describing nasal sensations. The terms describing sensations such as ' nasal sensation of airflow', ' nasal congestion' and 'nasal obstruction' are not clearly defined. The following definitions are put forward for discussion and in order to clarify the use of the terms in this review.

\section{Nasal sensation of airflow}

'Nasal sensation of airflow' is mainly related to the cooling action of the inspired air on cold receptors in the nose (6). 'Nasal sensation of airflow' can be scored by patients on a $100 \mathrm{~mm}$ visual analogue scale with the extremes labelled 'nose feels extremely clear' and 'nose feels extremely blocked' (6). This sensation has been shown to be influenced by menthol which is known to act on cold receptors in the nose.

\section{Nasal congestion}

'Nasal congestion' can be defined as the sensation of pressure related to the swelling of blood vessels lining the nasal passages. This sensation may be related to the stimulation of mechanoreceptors in the nose. Since nasal congestion is one of the causes of nasal obstruction it may be difficult to separate this sensation from the sensation of nasal obstruction.

\section{Nasal obstruction}

'Nasal obstruction' can be defined as the sensation of increased effort or change in airway pressure on inspiration when the nasal resistance is high or the nasal airway is occluded. This sensation may be related to the stimulation of muscle spindles and proprioceptors in the respiratory muscles of the diaphragm and chest wall. The sensation of nasal obstruction may also be related to stimulation of pressure receptors in the airway, as nasal and laryngeal pressure receptors responsive to negative pressure have been described in animal experiments $(1,7)$.

This review will discuss the factors that influence nasal sensations and try to explain the relationship between subjective and objective measures of nasal function.

\section{NASAL SENSATION AND NASAL SYMPTOMS}

Nasal symptoms such as itching are quite distinct and easily described by the patient and readily understood by the clinician. This is not the case with symptoms of nasal obstruction. In describing the nasal symptoms the patient is interested in those aspects that cause discomfort or disturbance and these may not equate to those aspects of the symptoms that are of interest to the clinician, such as the patency of the nose as determined by direct examination or by measurements of nasal airway resistance and minimum cross sectional area. The clinician is primarily concerned with functional and physical aspects of the nasal anatomy and physiology whereas the patient is concerned with sensations from the nose which are difficult to describe and even more difficult to locate.

There has been some progress in our understanding of this problem with the introduction of the concept of 'nasal sensation of airflow' and a standardised way of measuring this sensation using a visual analogue scale (8-11). However, there is still a great deal of work to do in this area, especially in trying to understand the relationships between physical changes in the nasal airway, the generation of symptoms, and how medical and surgical treatments may influence the sensation of nasal airflow.

\section{SENSATION OF NASAL AIRFLOW}

The concept of 'sensation of nasal airflow' is a relatively new concept. Early textbooks on the nose discuss the sensory innervation of the nose in relation to sneezing and itching but do not discuss the sensation of nasal airflow (12,). The first use of the term sensation of nasal airflow was in 1983 in relation to the effects of menthol and related compounds on the nose in healthy persons $(13,14)$.

\section{Anatomy and physiology}

The sensation of nasal airflow is perceived as a cool sensation. The cool sensation is related to the transfer of heat from the lining of the nose to the air stream. The loss of heat is determined by the temper- 
ature and humidity of the inspired air, with cold dry air causing the greatest heat loss and warm moist air providing little or no sensation.

The sensation of nasal airflow is mediated mainly by the trigeminal nerves supplying the nose. The nose, as the entrance to the respiratory system is strategically placed to sense the properties of the inspired air and the greatest cooling effect of the inspired air occurs at the entrance to the nose. The air is progressively warmed as it passes through the nose and the air stream is close to body temperature by the time it reaches the larynx. The larynx contains thermoreceptors which act as flow receptors (15) and although these may participate in the reflex control of breathing it is unlikely that they are involved in the sensation of airflow.

The anterior portion of the nose forms the nasal vestibule and this is lined with skin, almost as though a portion of the facial skin had been turned inwards to form the vestibule. At the entrance into the nares the skin is covered with short, stiff, hairs,or vibrissae, and these are better developed in the male than the female as their growth is stimulated by the male sex hormones just like the facial hair. The vibrissae are extremely sensitive to certain kinds of mechanical stimuli, and light displacement produces a sensation of itch or tickle which may cause sneezing. However, forceful inspiration and expiration which evenly displaces most of the vibrissae does not cause any appreciable sensation (16). The vibrissae lining the nasal vestibule do not appear to participate in the sensation of nasal airflow and their main role appears to be to form a screen or filter to protect the respiratory tract from inspiration of large particles and insects.

The nasal vestibule is lined with a stratified squamous epithelium which has sensory properties similar to those of the facial skin. Cauna (1982) (16) states that nasal thermoreceptors which detect nasal airflow are situated in the nasal vestibule. Since the facial skin has thermoreceptors that detect cooling it is reasonable to assume that they are also found in the skin lining the nasal vestibule. The nasal vestibule narrows at the entrance to the nasal cavity at the site of the nasal valve which forms the smallest crosssectional area of the whole respiratory tract.
The nasal valve area is strategically placed at the entrance of the nasal cavity and the inspired air velocity is maximal at this point. Any cooling or mechanical stimulus from the inspired air would be greatest at this point and the nasal valve area may be the major site for the origin of the sensation of nasal airflow.

Just posterior to the nasal valve the skin gradually changes into the ciliated respiratory nasal epithelium. The transition zone between the skin lining the vestibule and the ciliated respiratory epithelium is characterised by the presence of high and narrow dermal papillae, each of which is filled with a long capillary loop $(17,18)$. The function of these interesting papillae is not known but they may have a role as thermoreceptors as there is some evidence that cooling causes a change in blood flow through the capillary circulation (19).

The nasal vestibule and nasal valve may have the dominant sensory input for nasal sensation of airflow but one cannot exclude the respiratory epithelium from some contribution to the sensation of nasal airflow. The respiratory epithelium is supplied by sensory nerve fibres from maxillary branches of the trigeminal nerve and the epithelium is sensitive to mechanical and thermal stimuli. The skin lining the nasal vestibule is reported to be much more sensitive than the respiratory epithelium of the nasal cavity to mechanical and thermal stimuli applied by means of an air jet (20). One might expect that it would be possible to gather more information about the separate roles of the nasal vestibule and nasal cavity by using a local anaesthetic to eliminate the contribution of either the nasal vestibule or the respiratory epithelium. However, experiments using local anaesthetics have produced conflicting results and the only reasonable conclusion from these results at present is that both the skin of the nasal vestibule and the respiratory epithelium have a role in the sensation of nasal airflow (20-22).

\section{Measurement of sensation of nasal airflow}

The sensation of nasal airflow like any other sensation such as touch or temperature is something which we 'feel' and therefore can only be investigated by asking human volunteers to describe how they feel. 
Obviously, any type of animal experiment is not possible on the sensation of nasal airflow as animals cannot describe their feelings. The description of human sensations is limited by language as human volunteers must in some way describe their feelings in response to a question.

The first experiments on nasal sensation of airflow were conducted in 1983 and used simple questions, and a yes or no response, to determine if there were any changes in nasal sensation of airflow after exposure to menthol or exercise $(13,14)$. In 1987 a numerical scale was used for the first time to measure changes in nasal sensation to airflow in a study on the effects of menthol on the nose (23).

The $100 \mathrm{~mm}$ visual analogue scale was introduced as an instrument to measure nasal sensation of airflow in 1988 in a study on the effects of D and L isomers of menthol on nasal sensation of airflow (8). The $100 \mathrm{~mm}$ visual analogue scale or VAS labelled with extremes of sensation was first introduced into medicine by Aitken in 1964 who used the scale to investigate mood changes in airline pilots and the degree of asphyxia in volunteers breathing through mouth pieces of different resistance (24).

The VAS scale first used to study nasal sensation of airflow (8) was marked on either side 'extremely clear' and extremely blocked and this type of VAS has since been used in many similar experiments and has become a standard measure of nasal sensation of airflow (4, 25-27).

Subjective scores of nasal sensation of nasal airflow such as the VAS can be influenced by factors such as the way in which the scoring is conducted. The results of a study can easily be biased by the person conducting the scoring as the tone of their voice, their appearance etc. can influence the score. It is therefore essential that any instructions concerning the scoring are given to the patients in a standard way, preferably in the form of written instructions and under standardised conditions. If care is taken to ensure that both the investigator and patient are blinded to the study treatments etc. and the scoring method is standardis$\mathrm{ed}$, then the VAS is a reliable and reproducible measure of nasal sensation of airflow that can be used for research and clinical assessments.

\section{Factors that influence sensation of nasal airflow}

Since the sensation of nasal airflow is caused by the movement of air through the nasal passages one would predict that this sensation would be related to the nasal airway resistance. However, there is much controversy concerning the relationship between nasal airway resistance and nasal sensation of airflow and this will be discussed in detail below.

The sensation of nasal airflow is mediated by the trigeminal nerves supplying the nasal vestibule and respiratory epithelium, and damage to this nerve supply or anaesthetic block would be expected to cause a decreased sensation of nasal airflow. Local anaesthesia of the nasal vestibule by infiltration of lignocaine into the floor of the nasal vestibule has been reported to cause a sensation of nasal obstruction (21). Topical anaesthesia of the respiratory epithelium has not given such clear results as anaesthesia has been reported to cause both an increase (28) and a decrease in sensation of nasal airflow (22).

Medications containing menthol are widely used for the treatment of nasal congestion associated with upper respiratory tract infection. Studies on healthy volunteers and patients with nasal congestion have shown that inhalation of menthol vapour or ingestion of a lozenge containing menthol causes a subjective increase in the sensation of nasal airflow without any change in nasal airway resistance $(6,13,14,29)$. The results of one study investigating the effects of ingestion of a menthol lozenge on sensation of nasal airflow and nasal resistance to airflow in human volunteers with common cold are illustrated in Fig.1 (6).

Menthol causes a cool sensation in the nose by stimulation or sensitisation of cold receptors (29) and this is not related to any actual increase in nasal airflow through the nasal passages. However this is not a new concept since as early as 1886 Golsscheider (30) reported that menthol stimulated nerve 'endings' for cold, and in 1927 Fox (31) reported that topical application of menthol to the nasal epithelium caused a subjective sensation of nasal patency even though nasal airway resistance was actually increased.

The $\mathrm{L}$ isomer of menthol is much more potent than the $\mathrm{D}$ isomer of menthol in its cooling action and its effects on nasal sensation of airflow, and this may be 


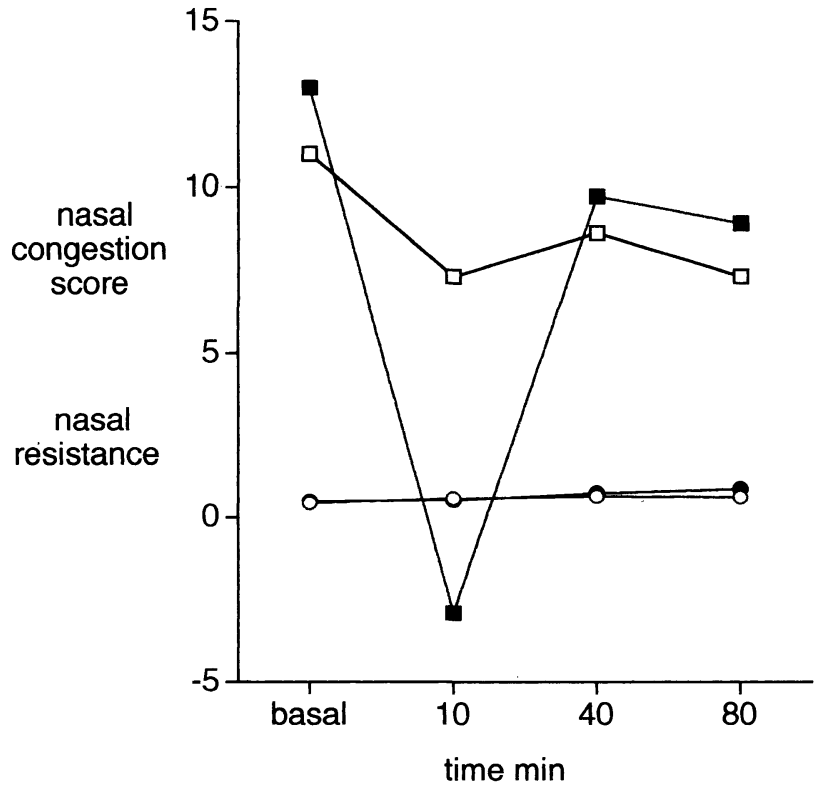

Figure 1 The effects of ingestion of an $11 \mathrm{mg} \mathrm{L-}$ menthol lozenge on subjective sensation of nasal congestion and nasal resistance to airflow in human volunteers with common cold. The subjective sensation of nasal congestion was significantly reduced $10 \mathrm{~min}$. after ingestion of the lozenge but nasal airway resistance as measured by rhinomanometry was unaffected. Shaded symbols represent the values for the menthol treated group $(n=30)$ and the open symbols represent the mean values for the placebo treated group $(n=32)$. Results taken from Eccles et al. (1990) (6).

explained by menthol having a specific pharmacological action on cold receptors in the nose $(8,9$, 29). The sensitising action of menthol on nasal cold receptors is believed to be due to a specific action of menthol on the calcium channels of the cold receptor $(29,32)$.

Patients with atrophic rhinitis often present with a symptom of decreased sensation of nasal airflow (often referred to in a very confusing way as a sensation of nasal obstruction) despite the fact that their nasal passages are abnormally patent due to loss of nasal turbinate tissue (33). The unusual nasal sensation in patients with atrophic rhinitis may be due to loss of sensation of nasal airflow due to atrophy of the nasal turbinates and gross loss of nasal tissue, including loss of sensory nerves supplying the respiratory epithelium.
Inflammation of the nasal epithelium due to infection and allergy causes the synthesis and release of chemical mediators such as histamine, prostaglandins, bradykinin and tachykinins. These mediators cause vasodilation of nasal blood vessels and nasal congestion but they may also cause a decrease in sensation of nasal airflow by influencing the activity of cold receptors in the nose since these mediators are known to interact with sensory nerve endings. For example, histamine interacts with sensory nerve endings in the nose to cause a sensation of itching, and histamine may also.inhibit the activity of cold receptors to cause a sensation of decreased nasal airflow. Even if mediators such as histamine do not have a direct action on cold receptors they could have indirect effects by causing localised oedema of the nasal epithelium.

Nasal surgery such as turbinectomy, diathermy and cryotherapy which is directed at improving nasal airflow may damage nasal sensory nerves and cause a decrease in the sensation of nasal airflow despite a physical increase in nasal airflow.

\section{RELATIONSHIP BETWEEN SUBJECTIVE AND OBJECTIVE MEASURES OF NASAL FUNCTION}

The first studies on nasal sensation of airflow were related to the effects of menthol on the nose and these clearly demonstrated that there was no relationship between the sensation of nasal airflow and nasal airway resistance $(6,13,31)$. Perhaps this finding should have alerted clinicians that there would not necessarily be any relationship between objective and subjective measures of nasal function in patients complaining of nasal obstruction. However, surgeons did expect that there should be a relationship between objective and subjective measures of nasal airflow and were surprised to find that there was no relationship between these measures of nasal airflow.

A major problem in understanding the validity of the studies comparing objective and subjective nasal measures is that there is often little or no description of the methods and the scales used for subjective scores. Similarly, it is often difficult to compare and criticise studies because of the use of different subjective scales. Simola (1997) (4) says that a $100 \mathrm{~mm}$ 
visual analogue scale was used to record nasal paten$c y$, but no details are given of the descriptors used on either end of the scale. This lack of precision when using subjective scales is typical of much of the literature in this area.

Several studies have clearly demonstrated that there is no correlation between total nasal airway resistance as measured by rhinomanometry and subjective scores $(10,25,34,35)$.

Studies using acoustic rhinometry have found no relationship between minimum cross sectional area and subjective scores when both nasal passages are assessed together $(27,35)$.

Studies looking at acute changes in nasal airflow have found that there is a correlation between the changes in total nasal airway resistance and nasal sensation of airflow when studying the effects of a topical nasal decongestant or the effects of histamine challenge $(4,10)$.

Those studies which have investigated the nose as two separate nasal passages and measured unilateral nasal airway resistance and unilateral subjective scores have found that there is a better correlation between objective and subjective measures of nasal airflow than found when the nose is treated as a single airway $(27,36)$. Similarly when patients are asked which side of the nose was more obstructed their responses correlated with the measurements of unilateral nasal airway resistance (37).

Why is there little or no correlation between objective and subjective measures of nasal airflow?

There is clearly no relation between the sensation of nasal airflow and nasal airway resistance when studying the effects of menthol on the nose as menthol sensitise or stimulates the cold receptors in the nose to cause an increased sensation of nasal airflow without any decongestant action and without any change in nasal airway resistance (29).

The lack of correlation between objective and subjective measures of nasal function in patients may be due to the assessment of the nose as a single airway rather than two separate nasal passages. A patient complaining of nasal congestion may have asym- metrical nasal airflow due to a deviated nasal septum or due to the spontaneous changes in nasal airflow associated with the nasal cycle. In this case the patient may complain of nasal congestion despite the fact that the total nasal airflow through the two nasal passages in parallel is within the normal range. The amplitude of the spontaneous changes in nasal airway resistance is increased during acute upper respiratory tract infection and one side of the nose may become blocked whilst the other side has a normal airflow (38). When a patient is asked to score their sensation of nasal airflow they give an overall impression of how they feel. If one side of the nose is blocked and the other side is clear they are more likely to score that they feel blocked.

Objective measures of nasal airflow such as rhinomanometry and acoustic rhinometry concentrate on the nasal valve area. The resistance to airflow through the nose is determined by the point of minimum cross sectional area and this is located at the nasal valve, just anterior to the tip of the inferior turbinate (39) (40). Nasal airway resistance as measured by rhinomanometry and minimum cross sectional area as measured by acoustic rhinometry therefore provide information mainly about the nasal valve region of the nose and this is affected by the congestion of the inferior turbinate. However, it is possible for other areas of the nose such as the ethmoid region to be congested with little change in the degree of congestion of the inferior turbinate. Some factors influencing the patients perception of nasal sensation of airflow are illustrated in Fig. 2. Swelling of the superior and middle turbinates may cause a sensation of nasal congestion that is not related to any change in nasal airway resistance. Similarly, congestion of the Eustachian tube and ostia of the paranasal sinuses may cause pressure changes in the middle ear and paranasal sinuses that are felt by the patient as a sensation of nasal congestion.

It is therefore not surprising that objective measures of nasal airflow do not correlate with the patients perception of nasal congestion and scores for nasal sensation of airflow. One would not expect objective measures of total nasal airflow to correlate with subjective scores of nasal airflow as the nasal 


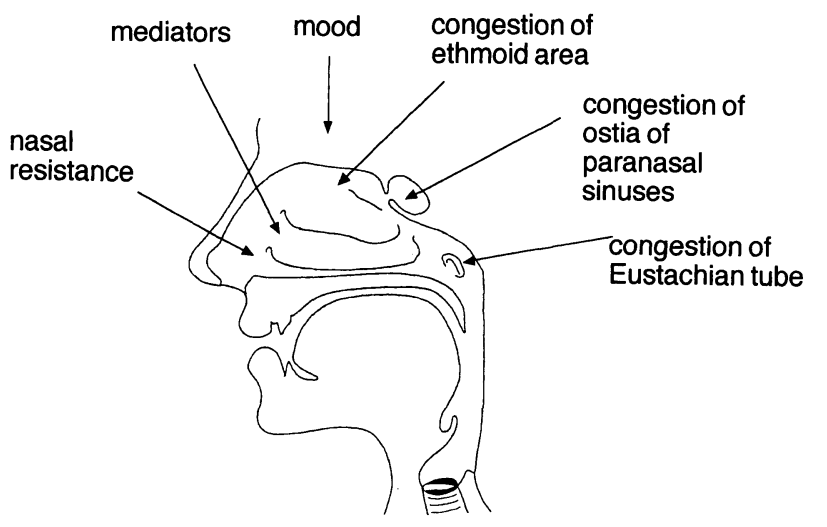

Figure 2 Some factors that influence the patients perception of sensation of nasal airflow; (nasal resistance) mainly determined by the minimum cross sectional area normally found at the level of the nasal valve; (mood) can affect subjective scores; (mediators) such as histamine may influence the activity of sensory nerves that detect airflow ; (congestion of the ethmoid area) gives a sensation of nasal congestion with little effect on nasal resistance to airflow ; (congestion of ostia of paranasal sinuses) causes pressure changes in the sinuses and the pressure sensations may be perceived as nasal congestion; (congestion of Eustachian tube) causes pressure changes in the middle ear that may be perceived as nasal congestion.

congestion is often asymmetrical with only one side of the nose having a decreased airflow. This may be why those studies which have investigated unilateral measures of nasal airflow and unilateral subjective scores have found some correlation between these measurements $(27,36,37)$, whereas those studies using total nasal airflow and an overall subjective score have failed to find any correlation between the measurements $(25,34,35)$.

The degree of correlation found between objective and subjective measures of nasal function is still very limited even in those studies using unilateral measures. Roithman et al. (27) found that the correlation coefficient for the relationship between unilateral objective and subjective measures varied from values of $r=0.22$ to $r=0.53$. It is useful to separate statistically significant correlations from clinically useful correlations as although a correlation value of $r=0.22$ may be statistically significant, this level of relation- ship between the objective and subjective measures of nasal function is not of any use to the clinician. With these low levels of correlation it would not be possible to predict the magnitude of the objective measurements form the subjective scores.

Perhaps, one of the more important reasons why there is much confusion in the relationship between objective and subjective scores of nasal function is that subjective scores have often been used carelessly and with little understanding of the relationship between nasal sensations and nasal symptoms.

\section{CONCLUSIONS}

There is much confusion concerning the use of subjective scores related to the symptom of nasal disease. A starting point for a better understanding of this area is a definition of terms such as ' nasal congestion', 'nasal obstruction' and 'nasal sensation of airflow' as given in this review. The use of the $100 \mathrm{~mm}$ visual analogue scale to measure nasal sensation of airflow has proved useful in research on the effects of menthol on the nose and these studies have demonstrated that there is no relation between the subjective and objective effects of menthol on the nose. Studies on patients have in general found little or no relationship between objective and subjective measures of nasal function when both nasal passages are considered together and only a poor relationship when the nasal passages are studied separately. Progress in this area is dependent on standardising the methods and terminology used for subjective measures of nasal function and a better understanding of the factors that influence the patients perception of nasal disease.

\section{REFERENCES}

1) Santambrogio G, Tsubone H, Santambrogio F B (1995) Sensory information from the upper airway-role in the control of breathing. Respiration Physiology 102: 1-16.

2) Widdicombe J G, Respiratory reflexes and defense, in Respiratory defense mechanisms, J.D. Brain, D.F. Proctor, and L.M. Reid, Editors. 1977, Marcel Dekker: New York. p. 593-630.

3) Widdicombe J (1996) Sensory mechanisms. Pulmonary Pharmacology $9:$ 383-387. 
4) Simola M, Malmberg H (1997) Sensation of nasal airflow compared with nasal airway resistance in patients with rhinitis. Clinical Otolaryngology $22: 260-262$.

5) Yaniv E, Hadar T, Shvero J, Raveh E (1997) Objective and subjective nasal airflow. American Journal of Otolaryngology 18 : 29-32.

6) Eccles R, Jawad M S, Morris S (1990) The effects of oral administration of $(-)$-menthol on nasal resistance to airflow and nasal sensation of airflow in subjects suffering from nasal congestion associated with the common cold. Journal of Pharmacy and Pharmacology 42 : 652-654.

7) Tsubone H (1990) Nasal pressure receptors. Japanese Journal of Veterinary Science 52 : 225232.

8) Eccles R, Griffiths D H, Newton C G, Tolley N S (1988) The effects of D and $L$ isomers of menthol upon nasal sensation of airflow. Journal of Laryngology and Otology 102 : 506-508.

9) Eccles R, Effects of menthol on nasal sensation of airflow, in Chemical Senses "Irritation "., B.G. Green, Editor. 1990, Dekker : New York. p. 275295.

10) Eccles $R$ (1990) Relationship between measured nasal airway resistance and the sensation of nasal airflow. Facial Plastic Surgery 7 : 278-282.

11) Eccles $R$ (1992) Nasal airway resistance and nasal sensation of airflow. Rhinology supplement $14: 86-90$.

12) Proetz A W, Essays on the applied physiology of the nose. Second edition ed. 1953, Saint Louis : Annals Publishing Co.

13) Eccles R, Jones A S (1983) The effect of menthol on nasal resistance to air flow. Journal of Laryngology and Otology 97 : 705-709.

14) Burrow A, Eccles R, Jones A S (1983) The effects of camphor, eucalyptus and menthol vapour on nasal resistance to airflow and nasal sensation. Acta Otolaryngologica (Stockholm) 96 : 157-161.

15) Sant'Ambrogio G, Mathew O P, Sant'Ambrogio $F$ B, Fisher J T (1985) Laryngeal cold receptors. Respiration Physiology 59 : 35-44.

16) Cauna N, Blood and nerve supply of the nasal lining, in The nose, upper airways physiology and the atmospheric environment, D.F. Proctor and I. Andersen, Editors. 1982, Elsevier: Amsterdam. p. 45-69.

17) Alverdes K (1930) Die Beziehungen der blutgefasse zum epithel im vestibulum nasi des menschen. Zeit Mikrosk Anat Forsch 22: 73-79.

18) Adams D R, Ireland W P (1990) Structure and organization of the subepithelial microvasculatur$\mathrm{e}$ in the canine nasal-mucosa. Microvascular Research 39 : 307-314.

19) Bari F, Ariwodola J O, Pleschka K (1995) Effects of calcium-channel blockers on cold-induced vasodilatation and elevated sympathetic tone in the canine internal maxillary artery bed. International Journal of Microcirculation-Clinical and Experimental 15: 92-103.

20) Clarke R W, Jones A S (1992) Nasal airflow receptors: the relative importance of temperature and tactile stimulation. Clinical Otolaryngology 17 : 388-392.

21) Jones A S, Crocher R, Wight R G, Lancer J M, Beckingham $E$ (1987) The effect of local anaesthesia of the nasal vestibule on nasal sensation of airflow and nasal resistance. Clinical Otolaryngology 12 : 461-464.

22) Eccles R, Morris S, Tolley N S (1988) The effects of nasal anaesthesia upon nasal sensation of airflow. Acta Otolaryngologica (Stockholm) 106: 152-155.

23) Eccles R, Lancashire B, Tolley N S (1987) Experimental studies on nasal sensation of airflow. Acta Otolaryngologica (Stockholm) 103 : 303306.

24) Aitken R C B (1969) Measurement of feelings using visual analogue scales. Proceedings of the Royal Society of Medicine 62 : 989-993.

25) Jones A S, Willatt D J, Durham L M (1989) Nasal airflow: resistance and sensation. Journal of Laryngology and Otology 103: 909-911.

26) Fairley J W, Durham L H, Ell S R (1993) Correlation of subjective sensation of nasal patency with nasal inspiratory peak flow-rate. Clinical Otolaryngology 18: 19-22.

27) Roithmann R, Cole P, Chapnik J, Barreto S M, Szalai J P, Zamel N (1994) Acoustic rhinometry, 
rhinomanometry, and the sensation of nasal patency -a correlative study. Journal of Otolaryngology 23 : 454-458.

28) Jones A S, Lancer J M, Shone G, Stevens J C (1986) The effect of lignocaine on nasal resistance and nasal sensation of airflow. Acta Otolaryngologica (Stockholm) 101 : 328-330.

29) Eccles $R$ (1994) Menthol and related cooling compounds. Journal of Pharmacy and Pharmacology 46 : 618-630.

30) Golscheider A (1886) Ueber specifische wirking des menthols. Archiv. fur Anatomie und Physiologische abeitlung Liepzig: 555-559.

31) Fox N (1927) Effect of camphor, eucalyptol, and menthol on the vascular state of the mucous membrane. Archives of Otolaryngology $6: 112-$ 122.

32) Schafer K, Braun H A, Isenberg C (1986) Effect of menthol on cold receptor activity. Journal of General Physiology 88 : 757-776.

33) Weir N, Golding-Wood D G, Infective rhinitis and sinusitis, in Scott-Brown's Otolaryngology, I.S. Mackay and T.R. Bull, Editors. 1997, Butterwoth-Heinemann: Oxford. p. pg 4/8/27.

34) Naito K, Cole P, Chaban R, Oprysk D (1988) Nasal resistance, sensation of obstruction, and rhinoscopic findings compared. American Journal of Rhinology 2: 65-69.
35) Tomkinson A, Eccles R (1996) Comparison of the relative abilities of acoustic rhinometry, rhinomanometry, and the visual analog scale in detecting change in the nasal cavity in a healthy adult-population. American Journal of Rhinology 10: 161-165.

36) Sipila J, Suonpaa J, Silvoniemi $P$, Laippala $P$ (1995) Correlations between subjective sensation of nasal patency and rhinomanometry in both unilateral and total nasal assessment. ORL-Journal For Oto-Rhino-Laryngology and its related specialties $57: 260-263$.

37) Sipila J, Suonpaa J, Laippala P (1994) Sensation of nasal obstruction compared to rhinomanometric results in patients referred for septoplasty. Rhinology 32 : 141-144.

38) Eccles R, Reilly M, Eccles K S J (1996) Changes in the amplitude of the nasal cycle associated with symptoms of acute upper respiratory tract infection. Acta Otolaryngologica (Stockholm) $116: 77-81$.

39) Haight J S J, Cole P (1983) Site and function of the nasal valve. Laryngoscope $93: 49-55$.

40) Eccles R, Evaluation of the nasal airway and nasal challenge, in Scott-Brown's Otolaryngology, I.S. Mackay and T.R. Bull, Editors. 1997, Butterwoth-Heinemann: Oxford. p. 4/4/1 - 4/4/ 15 